\title{
Dimethyl fumarate protects nucleus pulposus cells from inflammation and oxidative stress and delays the intervertebral disc degeneration
}

\author{
HAINIAN ZHU* , GANG CHEN* , YUHUA WANG, XUCHEN LIN, \\ JINGYUAN ZHOU, ZENGSHUN WANG and NANANGXIU SUO \\ Department of Orthopedics, Qinghai Provincial People's Hospital, Xining, Qinghai 810007, P.R. China
}

Received March 26, 2020; Accepted September 10, 2020

DOI: $10.3892 /$ etm.2020.9399

\begin{abstract}
Lower back pain is a common problem in middle-aged and elderly people, and intervertebral disc degeneration (IVDD) is often the main cause. The present study aimed to explore the effects of dimethyl fumarate (DMF) on inflammation and oxidative stress in the intervertebral disc. C57/BL6 mice were used to construct an IVDD model by tail suspension and daily intraperitoneal injections of $10 \mathrm{mg} / \mathrm{kg}$ DMF were administered to analyze the effects of DMF on IVDD. In addition, human nucleus pulposus (NP) cells were cultured and stimulated cells with recombinant human IL-1 $\beta$ and DMF to examine the effects of DMF on inflammation and oxidative stress in NP cells. DMF significantly increased the intervertebral disc height index of mice and inhibited the degradation of the extracellular matrix of mouse NP tissue. In addition, DMF also decreased the expression of inflammatory factors [including IL-6, IL-8, matrix metalloproteinase (MMP)3 and MMP13] in NP cells. In terms of oxidative stress, DMF significantly increased the antioxidative stress response in NP cells and reduced endoplasmic reticulum stress. DMF also increased the activity of the nuclear factor erythroid 2-related factor (Nrf) 2/heme oxygenase (HO)-1 signaling pathway in NP cells and increased the phosphorylation of Akt. DMF also increased the anti-inflammatory and antioxidative ability of NP cells by promoting the activity of the Nrf2/HO-1 and PI3K/Akt signaling pathways, thus delaying IVDD.
\end{abstract}

Correspondence to: Dr Nanangxiu Suo, Department of Orthopedics, Qinghai Provincial People's Hospital, 2 Gonghelu Road, Chengdong, Xining, Qinghai 810007, P.R. China

E-mail: suobo19910111@163.com

${ }^{*}$ Contributed equally

Key words: dimethyl fumarate, intervertebral disc degeneration, inflammation, oxidative stress

\section{Introduction}

The intervertebral disc is primarily divided into the peripheral fibrous annulus, the central nucleus pulposus (NP) and the upper and lower cartilage endplates. The surrounding fibrous rings are arranged according to concentric circles by multiple layers of fibrous cartilage and are supplied by vascular nutrients at the surface of the rings (1). The intervertebral disc is composed of glycoprotein, proteoglycan, collagen and elastic fibers. The proteoglycan in the intervertebral disc tissue can form a stable structure with elastin and collagen and adjust the pressure of the disc. The hydrating function of proteoglycan can maintain normal ion concentration and osmotic pressure in the intervertebral disc, so as to ensure the maintenance of the ion concentration and osmotic pressure gradient and the normal function of the intervertebral disc (2).

Lower back pain is a common problem in middle-aged and elderly people, and intervertebral disc degeneration (IVDD) is often the main cause. The prevalence of low back pain caused by IVDD reaches $20 \%$ worldwide, and $60 \%$ of people over 70 years old have been indicated to suffer from intervertebral disc disease (3). IVDD is caused by multiple factors, in which inflammation and oxidative stress play an important role (3). Studies have suggested that pro-inflammatory factors (such as TNF- $\alpha$ and IL-1 $\beta$ ) play an important role in the process of IVDD $(4,5)$. A significant increase in the expression of TNF- $\alpha$ and IL-1 $\beta$ was detected in the degenerated intervertebral disc of patients (6). These inflammatory factors can stimulate nerve growth and angiogenesis of NP cells by inducing the release of nerve growth factors, such as brain derived neurotrophic factor and vascular endothelial growth factor (7). In NP cells, TNF- $\alpha$ and IL-1 $\beta$ can also upregulate the expression of matrix metalloproteinases (MMPs) that degrade the extracellular matrix of NP cells (8). In addition, during the process NP cell degeneration, the balance of oxygen free radical production and antioxidant defense is disrupted, leading to the accumulation of reactive oxygen species (ROS), which further leads to cell damage and apoptosis (8). Therefore, inhibiting inflammation and apoptosis of NP cells is the key to preventing and treating IVDD.

Dimethyl fumarate (DMF) has been used in Europe to treat severe psoriasis. In 2012, the US Food and Drug 
Administration approved DMF application in the clinical treatment of relapse-remitting multiple sclerosis (9). In clinical statistical analysis, DMF has a good safety record so far in diseases such as psoriasis (10). DMF was superior to placebo in terms of the proportion of patients achieving a $\geq 75 \%$ improvement from baseline according to the Psoriasis Area and Severity Index (10). Studies have shown that DMF has immunomodulatory and anti-inflammatory effects in the treatment of neurological diseases, such as neuritis and spinal cord injury $(11,12)$. Therefore, some studies have applied DMF to different organs and different diseases. For example, in breast cancer cells, DMF can inhibit the NF- $\kappa \mathrm{B}$ pathway, thereby reducing the inflammatory response (13). After applying DMF to lung fibroblasts, it was reported that DMF inhibits the TNF- $\beta 1$ signaling pathway and ultimately exerts anti-fibrotic effects (14). It is noteworthy that numerous studies on the central nervous system have demonstrated that DMF can effectively activate nuclear factor erythroid 2-related factor (Nrf)2 and promote the activation of its downstream antioxidant stress pathway. Therefore, DMF has a good application prospect in terms of diseases associated with inflammation and oxidative damage (15). However, to the best of our knowledge, there is still no relevant study investigating whether DMF has anti-inflammatory and antioxidative effects in IVDD.

The present study used C57BL5 mice to construct an IVDD model, with the aim to study the effect of DMF on IVDD, and cultured human nucleus pulposus (NP) cells to study the molecular mechanisms underlying DMF function. The present results highlight the potential of DMF treatment for clinical IVDD.

\section{Materials and methods}

Animals and grouping. A total of 60 C57/BL6 male mice (Charles River Laboratories, Inc.) were used for the present study. The mice were 8 weeks old, weighed 20-25 g and were housed in standard barrier facilities $\left(25^{\circ} \mathrm{C} ; 55-65 \%\right.$ humidity; alternating light rhythm of $24 \mathrm{~h}$ ). The mice had free access to lab mouse food and distilled water, which were supplemented daily. The mice were randomly divided into three experimental groups (20 mice each): Control group, degenerative group and treatment group. Mice in the control group were routinely housed, while mice in the degenerative and treatment groups were modeled for IVDD. The mice in the treatment group were given a daily intraperitoneal injection of $10 \mathrm{mg} / \mathrm{kg} \mathrm{DMF}$ (Sigma-Aldrich; Merck KGaA) when the IVDD model was performed (16). The study was approved by The Animal Ethics Committee of Qinghai Provincial People's Hospital Animal Center (Xining, China; approval no. GS-XNH-17-A-0832).

IVDD model procedure and treatment. After anesthetizing the degenerative and treatment group mice with pentobarbital sodium at a dose of $40 \mathrm{mg} / \mathrm{kg}$, the tail of the mouse was wrapped with a medical tape and it was suspended on the top pulley in a special squirrel cage. The hind limbs of the mice are $>1-\mathrm{cm}$ above the ground and the forelimbs were able to crawl freely in the cages. The front paws could support the mice to move and feed (17). The health and behavior of the mice were observed twice a day. After 1 month of hanging the tail of the mouse, X-rays were used to observe the condition of the lumbar intervertebral disc. Signs of severe pain, including abnormal movement and sound, were considered humane endpoints requiring immediate euthanasia. All mice then received euthanasia via cervical dislocation (after being anesthetized using intraperitoneal administration of pentobarbital sodium at a dose of $40 \mathrm{mg} / \mathrm{kg}$ ). Death was confirmed by observing breathing and the heartbeat.

$X$-ray. After 1 month of hanging, the mice were anesthetized and placed in a tray in the right lateral position. Small animal $\mathrm{X}$-ray instruments were purchased from the American Faxitron and model number was MX-20. After obtaining X-ray images of each mouse, the intervertebral space height of the lumbar vertebrae of each mouse was measured, and the intervertebral disc height index (DHI) was calculated. DHI is the ratio of the sum of the heights of two adjacent vertebral bodies to the height of the intervertebral disc (18).

Cell culture and treatment. Human primary intervertebral disc nucleus cells were cultured to study the effect of DMF on IVDD. Human NP cells (cat. no. CP-H097) were purchased from Procell Life Science \& Technology Co., Ltd. NP cells were cultured using Dulbecco's modified eagle medium/F12 medium and added $10 \%$ fetal bovine serum (with $1 \%$ penicillin plus streptomycin) to the medium (all Gibco; Thermo Fisher Scientific, Inc.). The conditions of the cell culture incubator were set to $37^{\circ} \mathrm{C}$ and $5 \% \mathrm{CO}_{2}$. When the cell density reached $90 \%$, recombinant human IL-1 $\beta$ (50 ng/ml; Sigma-Aldrich; Merck KGaA) was used to stimulate NP cell degeneration. DMSO was used to dissolve the DMF powder and stored as a $10 \mu \mathrm{mol} / \mathrm{ml}$ stock solution. In total, $70 \mu \mathrm{mol} / 1$ of DMF was used to treat NP cells for $24 \mathrm{~h}$ at $37^{\circ} \mathrm{C}$.

Western blotting. At the end of the mouse modeling, the mice in all groups were sacrificed as aforementioned and the spine was extracted. Then, the intervertebral discs of the lumbar spine were scraped using a sterile blade and collected to extract the proteins using RIPA lysis buffer (Invitrogen; Thermo Fisher Scientific, Inc.). A BCA kit (Pierce; Thermo Fisher Scientific, Inc.) was used to measure protein concentration. An equal amount of protein $(30 \mu \mathrm{g})$ was added to each well of a $10 \%$ SDS-PAGE gel. After gel electrophoresis the proteins were transferred to polyvinylidene fluoride (PVDF) membranes (Roche Diagnostics). Then the membranes were blocked with a non-specific antigen with $5 \%$ skimmed milk for $1 \mathrm{~h}$ at room temperature. The following primary antibodies were incubated with the PVDF membranes: Collagen II (1:3,000; cat. no. ab34712) aggrecan (1:5,000; cat. no. ab3778), superoxide dismutase (SOD)1 (1:2,000; cat. no. ab183881), SOD2 (1:1,000; cat. no. ab68155), Peroxiredoxin 1 (Prdx1) (1:3,000; cat. no. ab41906) Prdx4 (1:4,000; cat. no. ab184167), MMP3 (1:5,000; cat. no. ab52915), MMP13 (1:3,000; cat. no. ab219620), C/EBP homologous protein (CHOP; 1:5,000; cat. no. ab179823), endoplasmic reticulum chaperone BiP (GRP-78; 1:2,000; cat. no. ab21685), caspase-12 (1:2,000; cat. no. ab62484), Nrf2 (1:3,000; cat. no. ab92946), HO-1, (1:2,000; cat. no. ab68477), Akt (1:1,000; cat. no. ab18785), phosphorylated Akt $(1: 3,000$; cat. no. ab38449) and $\beta$-actin (1:5,000; cat. no. ab6276) (all rabbit antibodies; Abcam) were used to incubate the PVDF membranes. CHOP, GRP-78 and 
Table I. Reverse transcription-quantitative PCR primer sequences.

A, Human

Name $\quad$ Sequence, $5^{\prime}-3^{\prime}$

IL-6

Forward

ACTCACCTCTTCAGAACGAATTG

Reverse

IL-8

$\begin{array}{ll}\text { Forward } & \text { CAGTTGAAGTTGCCATCAGC } \\ \text { Reverse } & \text { CAGTTGAAGTTACCATCAGC }\end{array}$

MMP3

Forward

Reverse

MMP13

Forward

Reverse

SOD1

Forward

Reverse

SOD2

Forward

Reverse

Prdx 1

Forward

Reverse

Prdx 4

Forward

Reverse

CHOP

Forward

Reverse

GRP-78

Forward

Reverse

Caspase-12

Forward

Reverse

Nrf2

\section{Forward}

Reverse

HO-1

Forward

Reverse

Akt

$\begin{array}{ll}\text { Forward } & \text { CCACAACAGCTATCGACCGTAC } \\ \text { Reverse } & \text { GTCATCTACTGACTGACACTA }\end{array}$

GAPDH

Forward

ACAACTTTGGTATCGTGGAAGG

Reverse

GGTCACCATCAACGCTGACTGACTG AGTCCGATCGACTACTAGCACTGACT

CGTACGTACTACTACGACGGCTAT GTGCACTGACGAGCACTGTGCGTTG

ATGGTGTTTGCAACCACATGGCATG

GACAACAGTTGGTTGCAACGGTCATG

ACTGAGCTCTGCATGACGTAGTC CGACTACGTACGACGCGACCTGCA

\section{CGCTGAGCTATGTTGCACACAGT} GCGTATGCGACTACACATCAGT

\section{GGTTGGTACACACATCAGCTACAC} GGTGTATCGACACTGTGTGACA

\section{AGTCTTGGTTGTGTACGTTGTG CGTGTGTGCACATACTGTGAC}

\section{GTGTGCACGACGTACAGCT} AGCTATCAGCACACGTTGTGT

GGTGTGCAGCACACGTACTGACG CGACTACACTGCACTGACTGAC

ACTGACGACTGACGTACGACGG GCTACGTACTGACTGATCCATGCA

AGCATCGTACTGACTGATGGTCAC GTGCTGACGTACTGACGACCATGC

B, Mouse

Collagen II

Forward ACGTGCGTATCACGGACGA Reverse
Table I. Continued.

\begin{tabular}{|c|c|}
\hline Name & Sequence, 5'-3' \\
\hline \multicolumn{2}{|l|}{ B, Mouse } \\
\hline \multicolumn{2}{|l|}{ Aggrecan } \\
\hline Forward & GGTGTTGACACACCGCGCGTATA \\
\hline Reverse & GTGCTCTATGAGATCTGATCG \\
\hline \multicolumn{2}{|l|}{ MMP3 } \\
\hline Forward & CGCTATTAGCGTCATCAGTC \\
\hline Reverse & GGTCTAGACTTAAAGCTACGT \\
\hline \multicolumn{2}{|l|}{ MMP13 } \\
\hline Forward & CAAGCTCGTTGTGCACGTGTA \\
\hline Reverse & CGTGTTGACACGTATGGTTCA \\
\hline \multicolumn{2}{|l|}{ GAPDH } \\
\hline Forward & GCATCACGTACTGACACCATG \\
\hline Reverse & CGACTGACTGACACTGCGA \\
\hline
\end{tabular}

caspase-12 are involved in the ER stress pathway (19). The PVDF membranes were incubated overnight at $4^{\circ} \mathrm{C}$. After washing the PVDF membranes with TBS-0.1\% Tween-20, the PVDF membranes were incubated with secondary HRP-goat anti-rabbit (1:3,000; cat. no. ab205718; Abcam) for $2 \mathrm{~h}$ at room temperature. Finally, the amount of protein expression was detected using ECL (Invitrogen; Thermo Fisher Scientific, Inc.). Protein extraction and detection from NP cells was also performed using this methodology.

$R N A$ isolation and reverse transcription-quantitative $(R T-q) P C R$. After obtaining mouse intervertebral disc tissue, total RNA was extracted using TRIzol ${ }^{\circledR}$ (Invitrogen; Thermo Fisher Scientific, Inc.). RNase-free water was used to dissolve RNA and the RNA concentration was detected using a spectrophotometer [Unico (Shanghai) Instrument Co., Ltd.; http://www.unicosh17.com.cn/]. SuperScript ${ }^{\circledR}$ IV Reverse Transcriptase (Invitrogen; Thermo Fisher Scientific, Inc.) was used to reverse transcribe the mRNA to cDNA $\left(10 \mathrm{~min}\right.$ at $55^{\circ} \mathrm{C}$; $10 \mathrm{~min}$ at $80^{\circ} \mathrm{C} ; 30 \mathrm{~min}$ at $4^{\circ} \mathrm{C}$ ) according to the manufacturer's protocol. Primers used to amplify cDNA were designed by Shanghai Jierui Biotechnology Co., Ltd. cDNA was amplified using PowerUp ${ }^{\mathrm{TM}} \mathrm{SYBR}^{\circledR}$ Green Master Mix (Invitrogen; Thermo Fisher Scientific, Inc.). The thermocycling conditions used were as follows: $2 \mathrm{~min}$ at $50^{\circ} \mathrm{C} ; 2 \mathrm{~min}$ at $95^{\circ} \mathrm{C}$; and 30 cycles of $3 \mathrm{sec}$ at $95^{\circ} \mathrm{C}$ and $30 \mathrm{sec}$ at $60^{\circ} \mathrm{C}$, according to the manufacturer's protocol. GAPDH expression was used as the control and the $2^{-\Delta \Delta \mathrm{Cq}}$ method was used to calculate relative RNA expression (20). The extraction and detection of RNA from NP cells was also performed using this methodology. The primers sequences are shown in Table I.

Cell Counting Kit (CCK)-8 assay. The optimal concentration of DMF and IL-1 $\beta$ was determined by detecting the viability of 
A

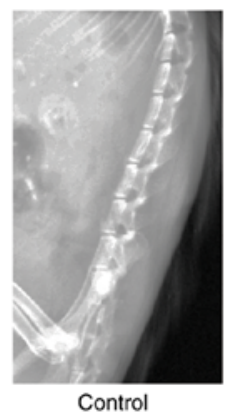

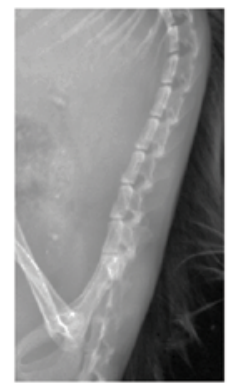

Tail suspension

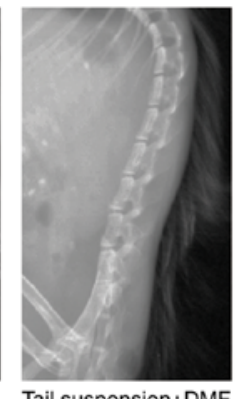

Tail suspension+DMF

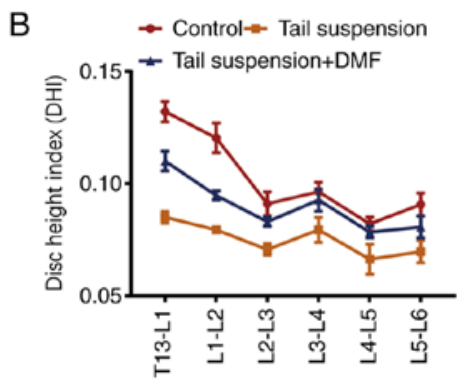

$\mathrm{E}$

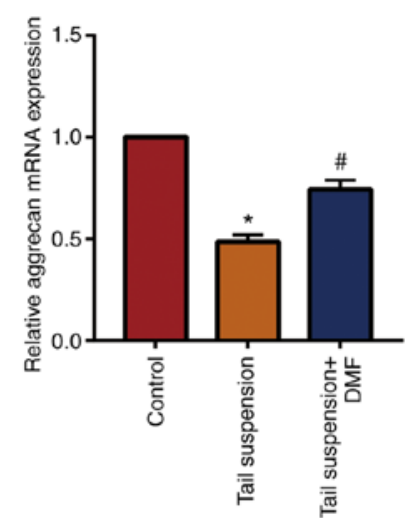

$\mathrm{F}$

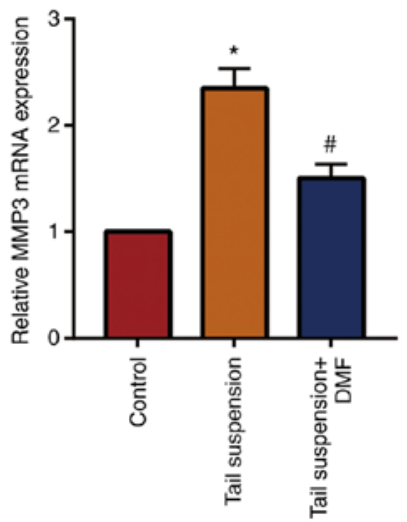

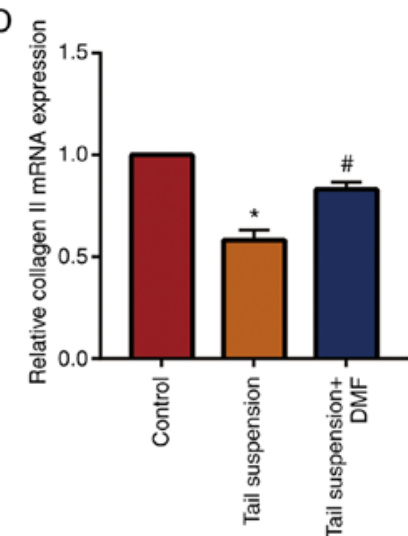

G

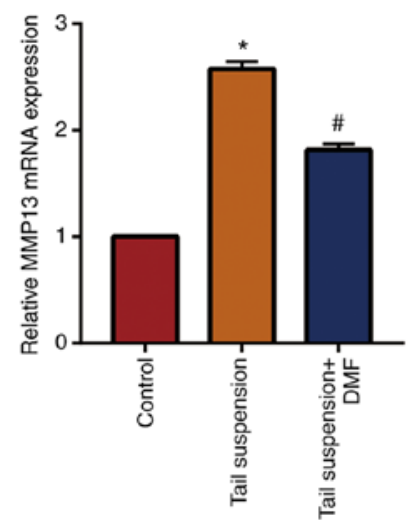

Figure 1. DMF relieves tail suspension-induced intervertebral disc degeneration in mice. (A) X-ray of mice intervertebral disc. (B) DHI of mice. (C) Western blot and (D-G) reverse transcription quantitative-PCR results of collagen II, aggrecan, MMP3 and MMP13. ${ }^{~} \mathrm{P}<0.05$ vs. the control group, ${ }^{~} \mathrm{P}<0.05$ vs. tail suspension group. DMF, dimethyl fumarate; DHI, disc height index; MMP, matrix metalloproteinase.

NP cells using CCK-8 assay, according to the manufacturer's protocol. The NP cells were plated onto 96-well plates, and then NP cells were stimulated with $10,30,50,70$ or $100 \mu \mathrm{mol} / 1$ DMF and 10, 30, 50, 70 or $100 \mathrm{ng} / \mathrm{ml}$ IL-1 $\beta$. After 3 days of stimulation, $10 \mu \mathrm{l} \mathrm{CCK-8} \mathrm{reagent} \mathrm{(Dojindo} \mathrm{Molecular}$ Technologies, Inc.) was added to each well and the cells were placed in the incubator for a further $4 \mathrm{~h}$. Then microplate reader was used to measure the absorbance at $450 \mathrm{~nm}$ of each well.

ELISA. After the density of NP cells reached 70\%, NP cells were stimulated with IL-1 $\beta(50 \mathrm{ng} / \mathrm{ml})$ alone of IL-1 $\beta$ $(50 \mathrm{ng} / \mathrm{ml})+\operatorname{DMF}(70 \mu \mathrm{mol} / \mathrm{l})$ for 3 days. At the end of the treatment, the supernatant of the cells was taken and the precipitate was removed using centrifugation $\left(10,500 \times \mathrm{g}, 15 \mathrm{~min}, 4^{\circ} \mathrm{C}\right)$. ELISA kits [Hangzhou Multi Sciences (Lianke) Biotech Co., Ltd.; http://lianke.Inxysf.com/introduce/] were used to detect the activity of IL-6 (cat. no. 70-EK106/2-96), IL-8 (cat. no. 70-EK108-96), MMP3 (cat. no. HA-ET1705-98-100) and MMP13 (cat. no. RK-KOA0275).

Detection of catalase (CAT) and SOD activity. The level of oxidative stress in NP cells was determined by detecting the activity of CAT and SOD in NP cells. After treating the NP cells for 1 day, the precipitate from the culture supernatant of the NP cells was removed using centrifugation. Then CAT (cat. no. 70-EK1306-24) and SOD (cat. no. 2A-706002-480) activity kits [both Hangzhou Multi Sciences (Lianke) Biotech Co., Ltd.] were used to measure the content of CAT and SOD.

Immunofluorescence (IF) staining. Plates (24-well) were used to culture NP cells. After the cell density reached $70 \%$, the cells were stimulated with IL-1 $\beta(50 \mathrm{ng} / \mathrm{ml})$ alone or IL-1 $\beta$ $(50 \mathrm{ng} / \mathrm{ml})+\operatorname{DMF}(70 \mu \mathrm{mol} / \mathrm{l})$ for 1 day at $37^{\circ} \mathrm{C}$. Then, the 

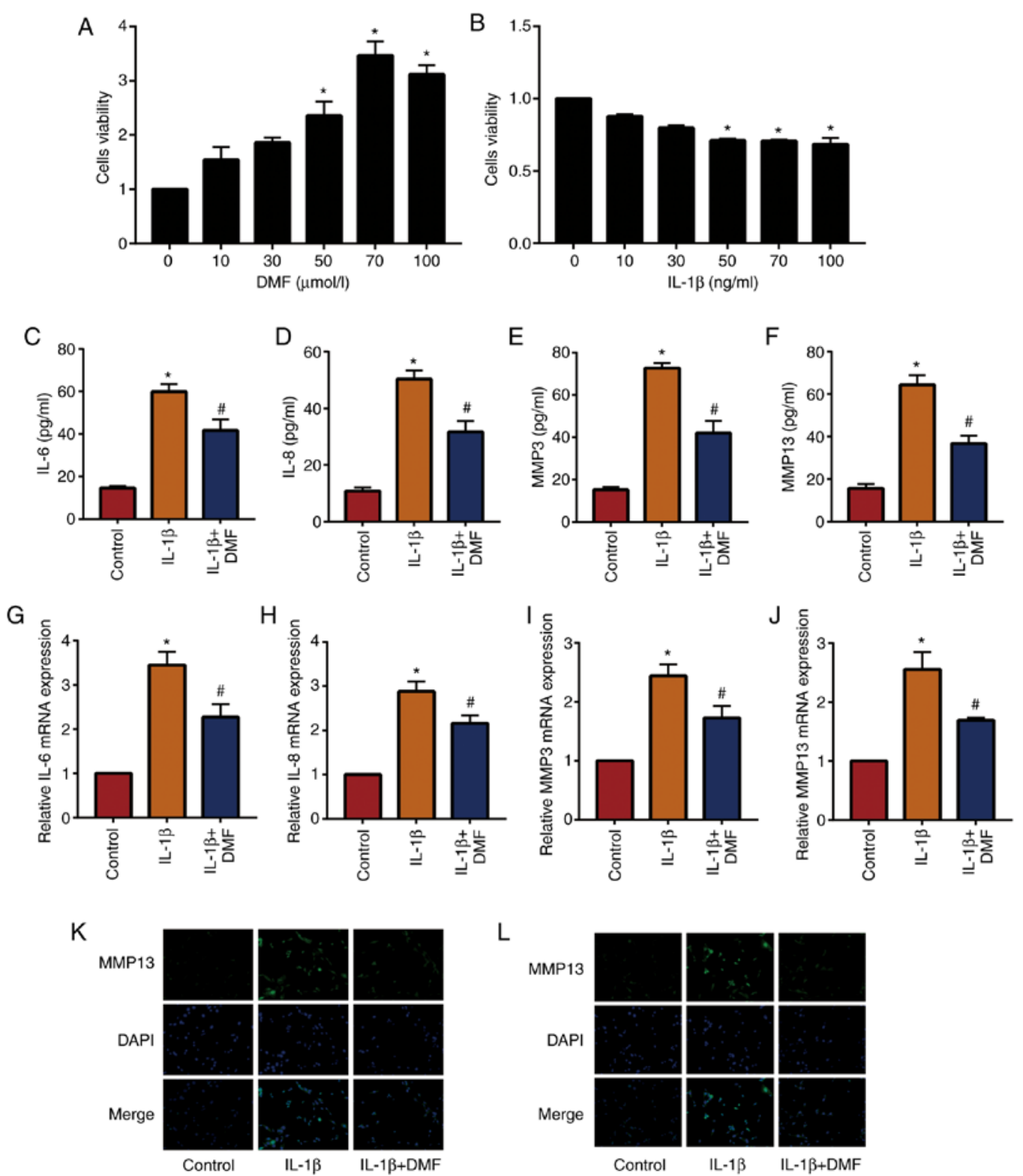

Figure 2. DMF reduces IL-1 $\beta$-induced inflammatory factor levels in nucleus pulposus cells. Cell Counting Kit-8 assay of (A) DMF and (B) IL-1 $\beta$. ELISA results of (C) IL-6, (D) IL-8, (E) MMP3 and (F) MMP13. (G-J) reverse transcription quantitative-PCR results of IL-6, IL-8, MMP3 and MMP13. Immunofluorescence staining and results of (K) MMP3 and (L) MMP13, magnification, 400x. ${ }^{*} \mathrm{P}<0.05$ vs. the control group, ${ }^{~} \mathrm{P}<0.05$ vs. the IL-1 $1 \beta$ group. DMF, dimethyl fumarate; MMP, matrix metalloproteinase.

medium was discarded, cells removed from the 24-well plates and treated with $4 \%$ paraformaldehyde and $0.5 \%$ Triton-PBS. Skimmed milk (5\%) was used to block non-specific antigens for $1 \mathrm{~h}$ at room temperature. Cells were incubated with the aforementioned primary antibodies at $4{ }^{\circ} \mathrm{C}$ overnight. After washing the cells with PBS, the cells were incubated with fluorescent secondary antibody dilution for $1 \mathrm{~h}$. DAPI was used to stain the nucleus for $10 \mathrm{~min}$ at room temperature. Finally, the staining results were observed using a fluorescence microscope (magnification, $\mathrm{x} 400$ ).

Statistical analysis. SPSS version 21.0 (IBM Corp) and GraphPad Prism version 7.0 (GraphPad Software) were used analyze the data. The data are presented as the mean \pm SD. All experiments were repeated more than three times. Comparisons between multiple groups performed using one-way ANOVA test followed by Tukey's post hoc test. $\mathrm{P}<0.05$ was considered to indicate a statistically significant difference.

\section{Results}

DMF relieves tail suspension-induced IVDD in mice. A mouse model of IVDD was established and DMF was administered to determine the effect of DMF on IVDD. X-ray results (Fig. 1A and B) showed that the DHI of the lumbar vertebrae of the tail-suspended mice was lower compared with that of the control group, while the DHI of the mice in the DMF group was significantly increased compared with the tail suspension group. These data indicated that DMF improved the intervertebral disc height of the lumbar vertebrae of the mice. Western blotting (Fig. 1C) and RT-qPCR (Fig. 1D-1G) showed that DMF significantly increased extracellular matrix collagen II 
A

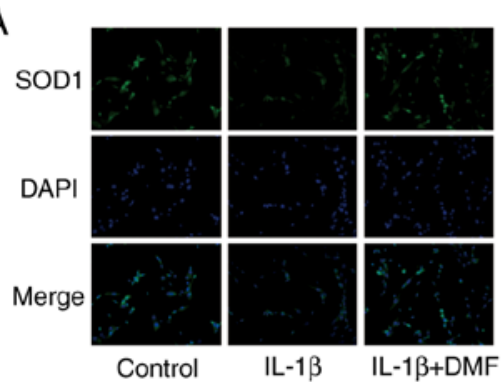

B

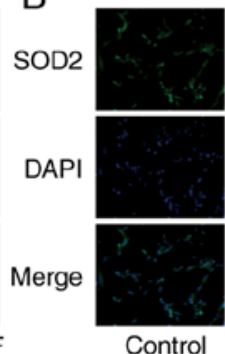

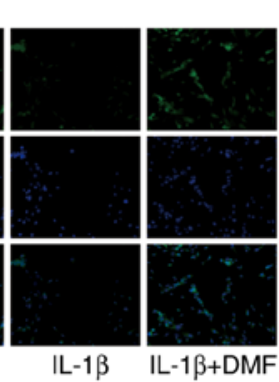

C

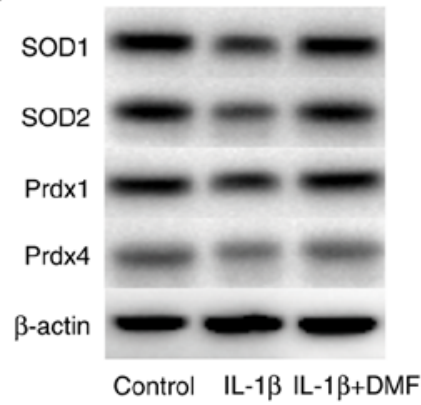

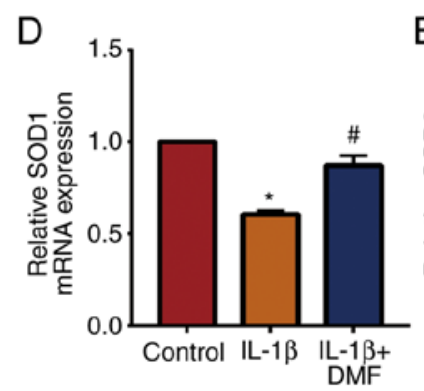
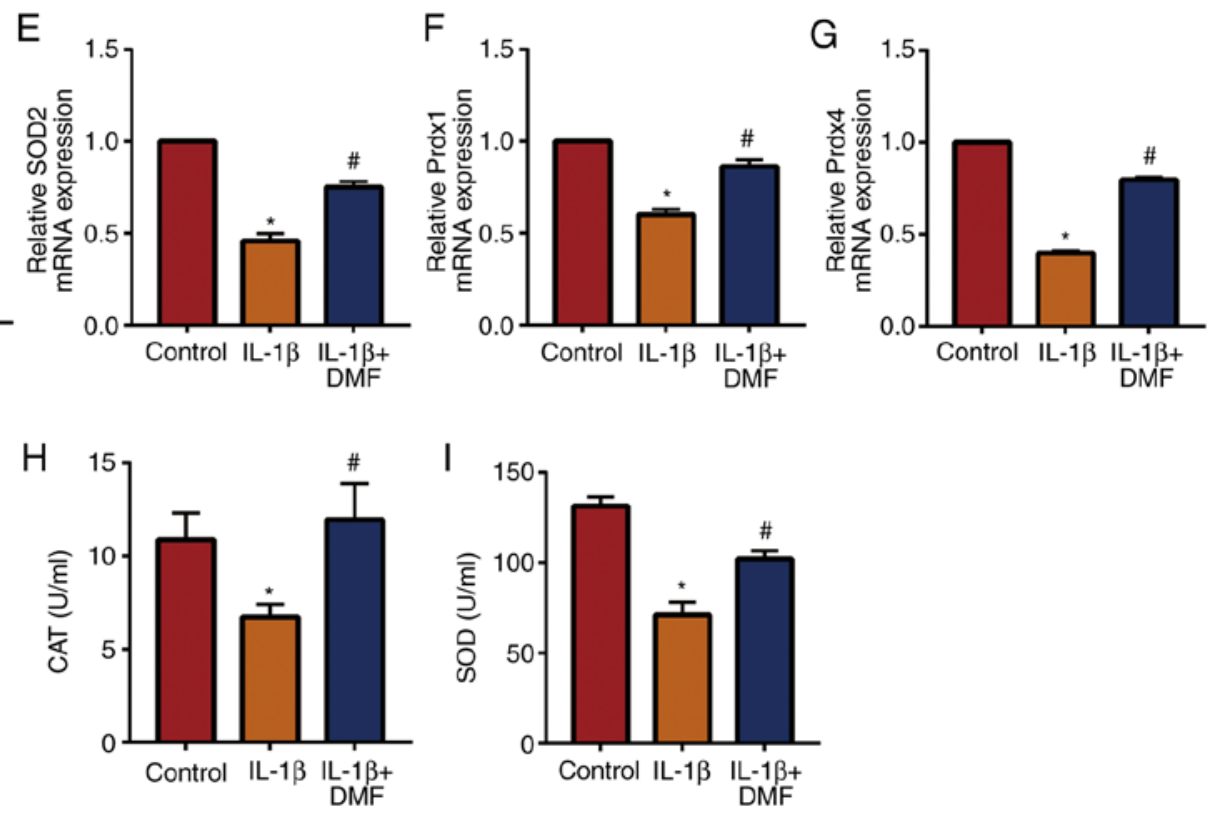

Figure 3. DMF inhibits oxidative stress in nucleus pulposus cells. Immunofluorescence staining results of (A) SOD1 and (B) SOD2 (magnification, 400x. (C) Western blot and reverse transcription quantitative-PCR results of (D) SOD1, (E) SOD2, (F) Prdx1 and (G) Prdx4. Activity of (H) CAT and (I) SOD. ${ }^{*} \mathrm{P}<0.05$ vs. the control group, ${ }^{\#} \mathrm{P}<0.05$ vs. the IL-1 $\beta$ group. DMF, dimethyl fumarate; SOD, superoxide dismutase; Prdx, peroxiredoxin 1; CAT, catalase.

and aggrecan in NP tissue, and significantly decreased MMP3 and MMP13 compared with the tail suspension group. These results indicated that the IVDD model was successfully established and that DMF alleviated IVDD in mice, likely due to the decreased degradation of the extracellular matrix of the NP tissue.

DMF decreases IL-1 $\beta$-induced inflammatory levels in $N P$ cells. In total, 10, 30, 50, 70 and $100 \mu \mathrm{mol} / 1 \mathrm{DMF}$ was used to stimulate NP cells and analyze cell viability using a CCK-8 assay (Fig. 2A). The results showed that cells stimulated with $70 \mu \mathrm{mol} / 1 \mathrm{DMF}$ reached the highest cell viability, therefore $70 \mu \mathrm{mol} / 1 \mathrm{DMF}$ was used to treat NP cells in subsequent experiments. In addition, $50 \mathrm{ng} / \mathrm{ml} \mathrm{IL}-1 \beta$ was the lowest dose to induce a significant difference compared with the untreated group (Fig. 2B). The results of ELISA (Fig. 2C-2F) and RT-qPCR (Fig. 2G-2J) showed that IL-1 $\beta$ increased the expression of inflammatory factors (IL-6, IL-8, MMP3 and MMP13) in NP cells compared with the control group, while IL-1 $\beta+$ DMF decreased these levels in NP cells compared with the IL-1 $\beta$ treatment group. IF staining (Fig. $2 \mathrm{~K}$ and L) also demonstrated that IL- $1 \beta+$ DMF decreased the expression of MMP3 and MMP13 compared with IL-1 $\beta$ only treatment.
DMF inhibits oxidative stress in NP cells. Oxidative stress is an important factor in IVDD (21), therefore the effect of DMF on oxidative stress in NP cells was analyzed. IF staining (Fig. 3A and B) detected the expression of SOD1 and SOD2, indicating that IL-1 $\beta+$ DMF treatment increased SOD1 and SOD2 expression compared with IL-1 $\beta$ treatment. The results of western blotting (Fig. 3C) and RT-qPCR (Fig. 3D-G) also showed that the expression of SOD1, SOD2, Prdx1 and Prdx4 was significantly decreased in IL-1 $\beta$-stimulated NP cells compared with the control group, while DMF attenuated IL-1 $\beta$-induced oxidative stress. In addition, the activity of CAT (Fig. 3H) and SOD (Fig. 3I) were examined in NP cells, and the results showed that IL-1 $\beta+$ DMF increased the activity of CAT and SOD compared with the IL- $1 \beta$ group.

DMF decreases $I L-1 \beta$-induced endoplasmic reticulum stress in NP cells. The expression of signaling molecules in the endoplasmic reticulum stress-related pathway was examined in NP cells. The results of IF staining (Fig. 4A and B) showed that the expression of CHOP and GRP-78 was increased in IL-1 $\beta$ induced NP cells and decreased by DMF. The results of western blotting (Fig. 4C) and RT-qPCR (Fig. 4D-4F) also showed that DMF decreased the expression of CHOP, GRP-78 
A

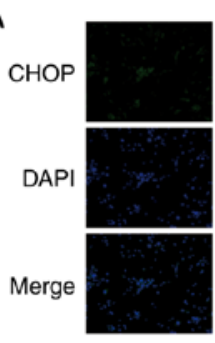

Control

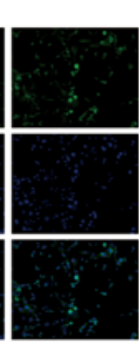

IL-1 $\beta$

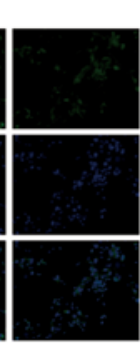

IL-1 $\beta+D M F$
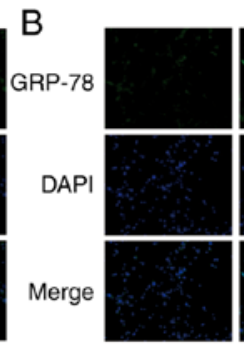

Control

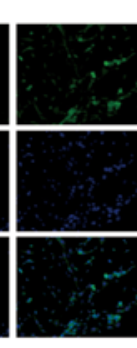

IL-1 $1 \beta$

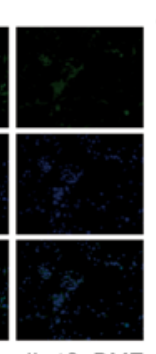

IL-1 $\beta+D M F$

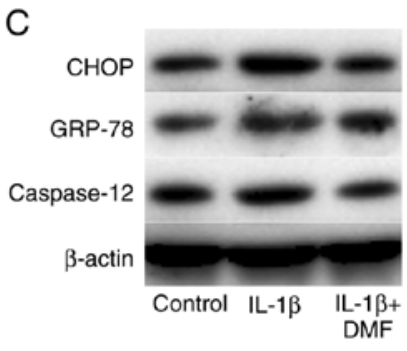

$\mathrm{F}$

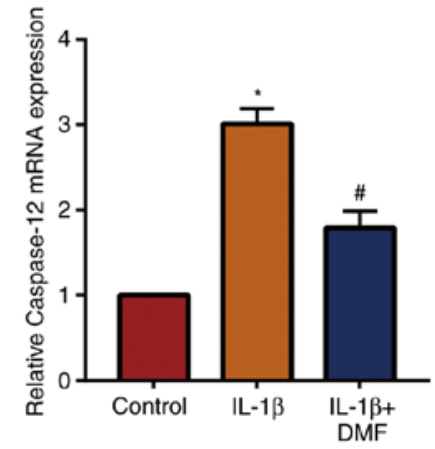

Figure 4. DMF decreases IL-1 $\beta$-induced endoplasmic reticulum stress in nucleus pulposus cells. Immunofluorescence staining results of (A) $\mathrm{CHOP}$ and (B) GRP-78 (magnification, 400x. (C) Western blot and reverse transcription quantitative-PCR results of (D) CHOP, (E) GRP-78 and (F) caspase-12. ${ }^{*}<<0.05$ vs. the control group, ${ }^{\sharp} \mathrm{P}<0.05$ vs. the IL- $1 \beta$ group. $\mathrm{DMF}$, dimethyl fumarate; CHOP, C/EBP homologous protein; GRP-78, endoplasmic reticulum chaperone BiP.

A

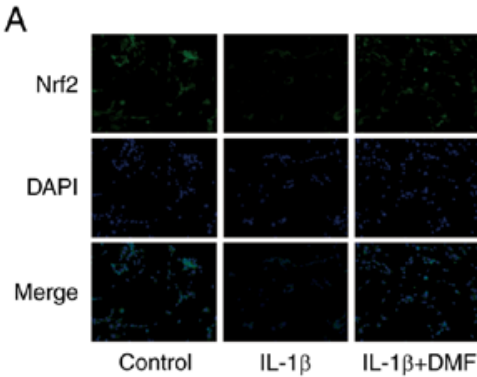

D

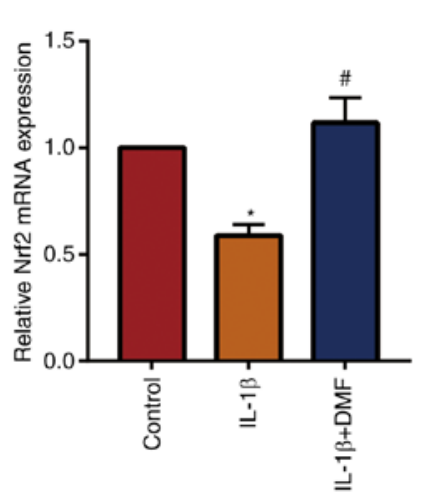

B

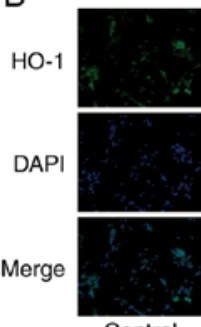

Control

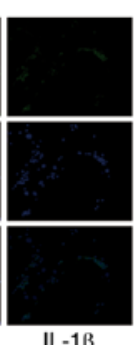

IL-1 $\beta$

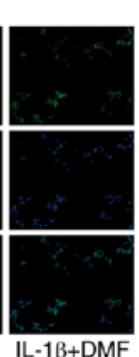

-1 $1 \beta+\mathrm{DMF}$

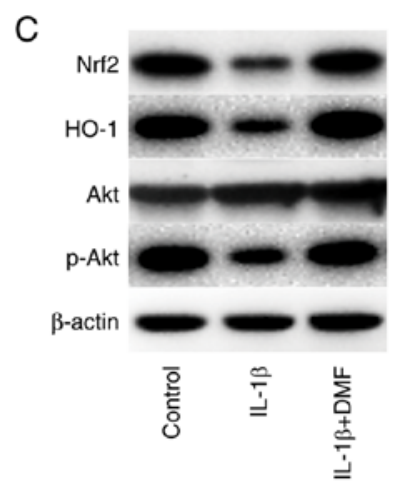

F
E

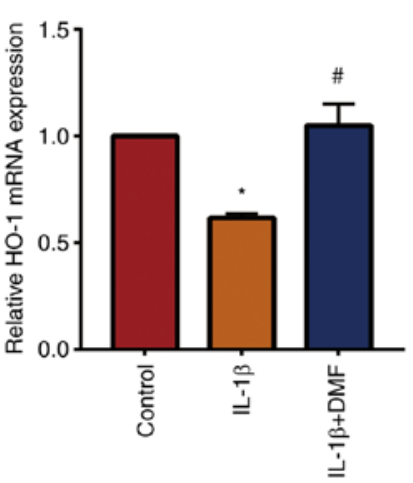

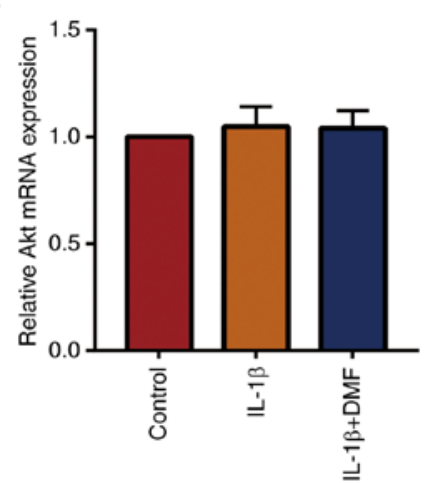

Figure 5. DMF promotes Nrf2/HO-1 and PI3K/Akt signaling pathways in nucleus pulposus cells. Immunofluorescence staining results of (A) Nrf2 and (B) HO-1 (magnification, 400x). (C) Western blot and reverse transcription quantitative-PCR results of (D) Nrf2, (E) HO-1 and (F) Akt. * $<<.05$ vs. the control group, ${ }^{\#} \mathrm{P}<0.05$ vs. the IL-1 $\beta$ group. DMF, dimethyl fumarate; Nrf2, erythroid 2-related factor; HO-1, heme oxygenase 1 ; p-, phosphorylated.

and caspase-12. These results indicated that DMF decreased endoplasmic reticulum stress levels in NP cells.

DMF promotes the Nrf2/HO-1 and PI3K/Akt signaling pathways in NP cells. The Nrf2/HO-1 and PI3K/AKT signaling pathways are important antioxidant and anti-inflammatory pathways (22). The results of IF staining (Fig. 5A and B) showed that DMF increased the expression of Nrf2 and HO-1 in NP cells compared with the control. Western blotting (Fig. 5C) and RT-qPCR (Fig. 5D-5F) also showed that DMF 
increased Nrf2 and HO-1 expression and promoted the phosphorylation of Akt compared with IL-1 $\beta$ treatment. These data indicated that DMF increased the activity of the Nrf2/HO-1 and PI3K/Akt signaling pathways.

\section{Discussion}

At present, the molecular mechanisms underlying degenerative disc disease are not fully understood. The pathological basis primarily involves inflammatory infiltration, decreased NP cell number and functional decline, NP dehydration, proteoglycan reduction and the change of collagen type and distribution (23). Gruber et al (24) reported that the number of aged and apoptotic NP cells in degenerated intervertebral disc tissue were increased, causing elevated inflammatory factors and abnormal metabolism of extracellular matrix (via increased MMP activity, and decreased quantity of collagen and proteoglycan). These changes are closely related to ROS. ROS are normal aerobic metabolites, including hydrogen peroxide, superoxide anion $\left(\mathrm{O}_{2}^{-}\right)$and hydroxyl radicals $(\mathrm{OH})$. Under normal circumstances, the production and elimination of ROS are in a dynamic equilibrium, and low concentrations of ROS function as signaling molecules in normal physiological activities, such as cell proliferation and differentiation, body damage repair and inflammatory immunity. When the ROS production in the body increases due to various reasons (such as injury and senescence) or the body's ability to resist ROS decreases, the body enters an oxidative stress state (25). At this time, ROS can directly cause the oxidative damage to DNA, proteins and lipids through the NP cell membrane, thereby causing destruction of these cells (26). High concentrations of ROS can also cause apoptosis in NP cells by disrupting the membrane permeability of mitochondria (27). The oxidative respiratory chain on the mitochondrial inner membrane is the primary site of ROS production, and it is also the most sensitive part of the ROS generation pathway (27). During the aerobic metabolism electron transfer process, some electrons will leak from the electron transport chain and combine with oxygen molecules to form $\mathrm{O}_{2}^{-}$(27). Importantly, mitochondria have a two-layer membrane structure; the outer membrane has good permeability, while the inner membrane has poor permeability, which prevents the transport of protons, most ions and water molecules (27). When the ROS content in the body exceeds the normal requirement, the mitochondrial membrane permeability transition pore is activated, increasing the permeability of the mitochondrial inner membrane, and the water molecules diffuse into the mitochondrial cytoplasm where there is a lower osmotic pressure (28). The increasing osmotic pressure of the mitochondria causes it to swell, leading to rupture of the mitochondrial outer membrane (29). Cytochrome $c$ located in the mitochondrial membrane gap is released into the cytosol to activate the downstream caspases 3, 6 and 7, causing apoptosis of the NP cells (29). In addition, ROS can also participate in the inflammatory response, regulate NP cell apoptosis and promote the synthesis and release of intracellular MMPs (MMPs 1, 3 and 13) and aggrecanase (ADAM-TS) by activating various pathways, such as JNK, p38/MAPK and NF- $\kappa B$ (29). MMPs and ADAM-TS are the main enzymes for extracellular matrix and proteoglycan and collagen II, which can induce the degradation of the intervertebral disc extracellular matrix, ADAM-TS-dependent proteoglycan and collagen II (30). This is the pathophysiological basis of the IVDD (30). It has been demonstrated that ROS participates in the occurrence and development of $\operatorname{IVDD}(31,32)$. In the present study, DMF-treated mice expressed less MMP3 and MMP13 in the intervertebral disc tissue, while the increased expression of extracellular matrix components (collagen II and aggrecan) indicated that DMF protected the extracellular matrix of NP cells from degradation of MMPs. In addition, DMF stimulated NP cells and significantly decreased inflammation and oxidative stress in NP cells, as shown by the decrease of inflammatory factor expression and increase of SOD activity. This indicated that the anti-inflammatory and antioxidative effects of DMF can protect NP cells. DMF was also reported to reduce IL-1 $\beta$-induced cell nucleus endoplasmic reticulum stress, which is further evidence of the protective effect of DMF on cell nucleus.

When various external factors, such as injury and infection among others, cause the balance between ROS production and the antioxidant defense system to be disrupted, the body enters an oxidative stress state, activating a series of antioxidant stress defense responses and antioxidant transcription factors, including Nrf2 and AP-1. Activation of these transcription factors ultimately increases the expression and synthesis of antioxidant enzymes, such as SOD, CAT and HO-1, which protects the body from oxidative stress (33). Nrf2 is an important antioxidant defense factor in the body, expressed in almost all cells. When the body's ROS levels increase, the Nrf2 located in the cytosol is dissociates from its inhibitor Keap1 and is activated (34). Free Nrf2 enters the nucleus and binds to the Maf protein to form a dimer and binds to the antioxidant response element, thereby initiating the transcriptional expression of a series of antioxidant enzymes (35). The Nrf2/HO-1 signaling pathway is a crucial protective pathway against oxidative stress caused by various external causes, such as injury and infection. Studies have confirmed that Nrf2/HO-1 signaling pathway can regulate $>200$ antioxidant proteases, phase II detoxification enzymes and anti-inflammatory factors $(36,37)$. The transcriptional expression of endogenous protective genes plays an irreplaceable role in antioxidative stress, anti-inflammatory, anti-apoptosis and antitumor responses (38). Studies have confirmed that numerous diseases are related to the increase of oxygen free radicals in the body, such as Alzheimer's disease, Parkinson's disease and pulmonary fibrosis, and there are several changes in the expression of Nrf2 at different stages of such diseases $(39,40)$. The degenerative disease of the intervertebral disc also has an inflammatory reaction accompanied by the pathological increase of oxygen radicals in the body (41). The role of Nrf2 in antioxidation and anti-inflammation has been recognized in a number of diseases, such as myocardial infarction and inflammatory bowel disease $(42,43)$; however, and whether these functions of Nrf2 are also closely associated with degenerative diseases of intervertebral disc is unclear. The PI3K/Akt pathway regulates apoptosis during oxidative stress. Studies have shown that activation of the PI3K/Akt pathway protects nerve cells from oxidative stress $(44,45)$. Inhibition of Akt activity makes cells more sensitive to ROS and more susceptible to oxidative damage (46). The PI3K/Akt signaling pathway plays an 
important role in the expression of antioxidant enzymes, including HO-1 and glutamate cysteine ligase. Therefore, Akt-related signaling pathways are important for the body to resist oxidative stress (47). In the present study, the activity of the Nrf2/HO-1 signaling pathway and the PI3K/Akt signaling pathway in the NP was significantly decreased after stimulation of the NP cells with IL-1 $\beta$. In DMF-stimulated NP cells, the expression of Nrf2 and HO-1 and the level of phosphorylated Akt was increased, indicating that DMF promoted the activity of these two signaling pathways in NP cells. This may be the mechanism of the anti-inflammatory and antioxidant effects of DMF in NP cells.

In DMF-treated mice, DHI was significantly increased and extracellular matrix degradation was also inhibited, indicating that DMF relieved IVDD in mice. In cell experiments, DMF increased the viability of NP cells and inhibited IL-1 $\beta$-induced inflammation, oxidative stress and endoplasmic reticulum stress. In addition, DMF also promoted the activity of the Nrf2/HO-1 and PI3K/Akt signaling pathways in NP cells, which helps clarify the mechanism of DMF in protecting intervertebral discs. Overall, DMF demonstrated good efficacy in protecting against IVDD. To the best of our knowledge, the present study is the first to report that DMF improves IVDD, and these results may aid in the development of DMF treatment to clinically prevent IVDD.

\section{Acknowledgements}

Not applicable.

\section{Funding}

No funding was received.

\section{Availability of data and materials}

All data generated or analyzed during this study are included in this published article.

\section{Authors' contributions}

HZ, GC and NS designed the study and performed the experiments. HZ and YW established the animal models. GC and XL collected the data. JZ and ZW analyzed the data. HZ, GC and NS prepared the manuscript. All authors read and approved the final manuscript.

\section{Ethics approval and consent to participate}

The present study was approved by The Animal Ethics Committee of Qinghai Provincial People's Hospital Animal Center (Xining, China; approval no. GS-XNH-17-A-0832).

\section{Patient consent for publication}

Not applicable.

\section{Competing interests}

The authors declare that they have no competing interests.

\section{References}

1. Wu CZ, Ou DQ, Rong LM, Xu YC, Dong JW, Fan L and Wang QY: Expression of lamin A/C protein in degenerated human intervertebral disc. Eur Rev Med Pharmacol Sci 22: 7607-7613, 2018.

2. González Martínez E, García-Cosamalón J, Cosamalón-Gan I, Esteban Blanco M, García-Suarez O and Vega JA: Biology and mechanobiology of the intervertebral disc. Neurocirugia (Astur) 28: 135-140, 2017 (In Spanish).

3. Navone SE, Marfia G, Giannoni A, Beretta M, Guarnaccia L, Gualtierotti R, Nicoli D, Rampini P and Campanella R: Inflammatory mediators and signalling pathways controlling intervertebral disc degeneration. Histol Histopathol 32: 523-542, 2017.

4. Lin X and Lin Q: MiRNA-495-3p attenuates TNF- $\alpha$ induced apoptosis and inflammation in human nucleus pulposus cells by targeting IL5RA. Inflammation 43: 1797-1805, 2020.

5. He M, Pang J, Sun H, Zheng G, Lin Y and Ge W: P14ARF inhibits regional inflammation and vascularization in intervertebral disc degeneration by upregulating TIMP3. Am J Physiol Cell Physiol 318: C751-C761, 2020.

6. Wang Y, Che M, Xin J, Zheng Z, Li J and Zhang S: The role of IL-1 $\beta$ and TNF- $\alpha$ in intervertebral disc degeneration. Biomed Pharmacother 131: 110660, Aug 24, 2020 (Online ahead of print).

7. Dowdell J, Erwin M, Choma T, Vaccaro A, Iatridis J and Cho SK: Intervertebral disk degeneration and repair. Neurosurgery 80: S46-S54, 2017.

8. Jiang JY and Lu XH: Biological treatment for intervertebral disc degeneration. Zhongguo Gu Shang 29: 576-580, 2016 (In Chinese).

9. Kalincik T, Kubala Havrdova E, Horakova D, Izquierdo G, Prat A, Girard M, Duquette P, Grammond P, Onofrj M, Lugaresi A, et al. Comparison of fingolimod, dimethyl fumarate and teriflunomide for multiple sclerosis. J Neurol Neurosurg Psychiatry 90: 458-468, 2019.

10. Blair HÄ: Dimethyl fumarate: A review in moderate to severe plaque psoriasis. Drugs 78: 123-130, 2018.

11. Hayashi G, Jasoliya M, Sahdeo S, Saccà F, Pane C, Filla A, Marsili A, Puorro G, Lanzillo R, Brescia MV and Cortopassi G: Dimethyl fumarate mediates Nrf2-dependent mitochondrial biogenesis in mice and humans. Hum Mol Genet 26: 2864-2873, 2017.

12. Oey O, Rao P, Luciuk M, Mannix C, Rogers NM, Sagar P, Wong A and Rangan G: Effect of dimethyl fumarate on renal disease progression in a genetic ortholog of nephronophthisis. Exp Biol Med (Maywood) 243: 428-436, 2018.

13. Al-Jaderi $Z$ and Maghazachi AA: Utilization of dimethyl fumarate and related molecules for treatment of multiple sclerosis, cancer, and other diseases. Front Immunol 7: 278, 2016.

14. Saidu NE, Noé G, Cerles O, Cabel L, Kavian-Tessler N, Chouzenoux S, Bahuaud M, Chéreau C, Nicco C, Leroy K, et al: Dimethyl fumarate controls the NRF2/DJ-1 axis in cancer cells: Therapeutic applications. Mol Cancer Ther 16: 529-539, 2017.

15. Krishnamoorthy S, Pace B, Gupta D, Sturtevant S, Li B, Makala L, Brittain J, Moore N, Vieira BF, Thullen T, et al: Dimethyl fumarate increases fetal hemoglobin, provides heme detoxification, and corrects anemia in sickle cell disease. JCI Insight 2: 96409, 2017.

16. Iniaghe LO, Krafft PR, Klebe DW, Omogbai EKI, Zhang JH and Tang J: Dimethyl fumarate confers neuroprotection by casein kinase 2 phosphorylation of Nrf2 in murine intracerebral hemorrhage. Neurobiol Dis 82: 349-358, 2015.

17. Che H, Li J, Li Y, Ma C, Liu H, Qin J, Dong J, Zhang Z, Xian CJ, Miao D, et al: p16 deficiency attenuates intervertebral disc degeneration by adjusting oxidative stress and nucleus pulposus cell cycle. Elife 9: e52570, 2020

18. Urquhart DM, Kurniadi I, Triangto K, Wang Y, Wluka AE, O'Sullivan R, Jones G and Cicuttini FM: Obesity is associated with reduced disc height in the lumbar spine but not at the lumbosacral junction. Spine (Phila Pa 1976) 39: E962-E966, 2014.

19. Rana SVS: Endoplasmic reticulum stress induced by toxic elements-a review of recent developments. Biol Trace Elem Res 196: 10-19, 2020.

20. Livak KJ and Schmittgen TD: Analysis of relative gene expression data using real-time quantitative PCR and the 2(-Delta Delta C(T)) method. Methods 25: 402-408, 2001. 
21. Xu WN, Zheng HL, Yang RZ, Liu T, Yu W, Zheng XF, Li B Jiang SD and Jiang LS: Mitochondrial NDUFA4L2 attenuates the apoptosis of nucleus pulposus cells induced by oxidative stress via the inhibition of mitophagy. Exp Mol Med 51: 1-16, 2019.

22. Loboda A, Damulewicz M, Pyza E, Jozkowicz A and Dulak J: Role of Nrf2/HO-1 system in development, oxidative stress response and diseases: An evolutionarily conserved mechanism. Cell Mol Life Sci 73: 3221-3247, 2016.

23. Centeno C, Markle J, Dodson E, Stemper I, Williams CJ, Hyzy M, Ichim $\mathrm{T}$ and Freeman M: Treatment of lumbar degenerative disc disease-associated radicular pain with culture-expanded autologous mesenchymal stem cells: A pilot study on safety and efficacy. J Transl Med 15: 197, 2017.

24. Gruber HE, Hoelscher GL, Ingram JA, Bethea S and Hanley EN Jr: Autophagy in the degenerating human intervertebral disc: In vivo molecular and morphological evidence, and induction of autophagy in cultured annulus cells exposed to proinflammatory cytokines-implications for disc degeneration. Spine (Phila Pa 1976) 40: 773-782, 2015.

25. Yang S and Lian G: ROS and diseases: Role in metabolism and energy supply. Mol Cell Biochem 467: 1-12, 2020.

26. Feng C, Yang M, Lan M, Liu C, Zhang Y, Huang B, Liu H and Zhou Y: ROS: Crucial intermediators in the pathogenesis of intervertebral disc degeneration. Oxid Med Cell Longev 2017: 5601593, 2017.

27. Nakamura T, Naguro I and Ichijo H: Iron homeostasis and iron-regulated ROS in cell death, senescence and human diseases. Biochim Biophys Acta Gen Subj 1863: 1398-1409, 2019

28. Nasto LA, Robinson AR, Ngo K, Clauson CL, Dong Q, St CC Sowa G, Pola E, Robbins PD, Kang J, et al: Mitochondrial-derived reactive oxygen species (ROS) play a causal role in aging-related intervertebral disc degeneration. J Orthop Res 31: 1150-1157, 2013.

29. Jiang LB, Cao L, Ma YQ, Chen Q, Liang Y, Yuan FL, Li XL, Dong $\mathrm{J}$ and Chen N: TIGAR mediates the inhibitory role of hypoxia on ROS production and apoptosis in rat nucleus pulposus cells. Osteoarthritis Cartilage 26: 138-148, 2018.

30. Ma KG, Shao ZW, Yang SH, Wang J, Wang BC, Xiong LM, Wu Q and Chen SF: Autophagy is activated in compression-induced cell degeneration and is mediated by reactive oxygen species in nucleus pulposus cells exposed to compression. Osteoarthritis Cartilage 21: 2030-2038, 2013.

31. Song Y, Wang Z, Liu L, Zhang S, Zhang H and Qian Y: 1,4-Dihydropyridine (DHP) suppresses against oxidative stress in nucleus pulposus via activating sirtuin-1. Biomed Pharmacother 121: 109592, 2020.

32. He R, Cui M, Lin H, Zhao L, Wang J, Chen S and Shao Z: Melatonin resists oxidative stress-induced apoptosis in nucleus pulposus cells. Life Sci 199: 122-130, 2018.

33. Fang W, Zhou X, Wang J, Xu L, Zhou L, Yu W, Tao Y, Zhu J, $\mathrm{Hu}$ B, Liang C, et al: Wogonin mitigates intervertebral disc degeneration through the Nrf2/ARE and MAPK signaling pathways. Int Immunopharmacol 65: 539-549, 2018.
34. Guo Z and Mo Z: Keap1-Nrf2 signaling pathway in angiogenesis and vascular diseases. J Tissue Eng Regen Med 14: 869-883, 2020.

35. Dimozi A, Mavrogonatou E, Sklirou A and Kletsas D: Oxidative stress inhibits the proliferation, induces premature senescence and promotes a catabolic phenotype in human nucleus pulposus intervertebral disc cells. Eur Cell Mater 30: 89-102, 2015.

36. Kasai S, Shimizu S, Tatara Y, Mimura J and Itoh K: Regulation of Nrf2 by mitochondrial reactive oxygen species in physiology and pathology. Biomolecules 10: 320, 2020.

37. Sajadimajd S and Khazaei M: Oxidative stress and cancer: The role of Nrf2. Curr Cancer Drug Targets 18: 538-557, 2018.

38. Panieri E, Buha A, Telkoparan-Akillilar P, Cevik D, Kouretas D, Veskoukis A, Skaperda Z, Tsatsakis A, Wallace D, Suzen S and Saso L: Potential applications of NRF2 modulators in cancer therapy. Antioxidants (Basel) 9: 193, 2020

39. Cherif H, Bisson DG, Jarzem P, Weber M, Ouellet JA and Haglund L: Curcumin and o-vanillin exhibit evidence of senolytic activity in human IVD cells in vitro. J Clin Med 8: 433, 2019.

40. Ungvari Z, Tarantini S, Nyúl-Tóth Á, Kiss T, Yabluchanskiy A, Csipo T, Balasubramanian P, Lipecz A, Benyo Z and Csiszar A: Nrf2 dysfunction and impaired cellular resilience to oxidative stressors in the aged vasculature: From increased cellular senescence to the pathogenesis of age-related vascular diseases. Geroscience 41: 727-738, 2019.

41. Feng C, Liu H, Yang M, Zhang Y, Huang B and Zhou Y: Disc cell senescence in intervertebral disc degeneration: Causes and molecular pathways. Cell Cycle 15: 1674-1684, 2016.

42. Strom J and Chen QM: Loss of Nrf2 promotes rapid progression to heart failure following myocardial infarction. Toxicol Appl Pharmacol 327: 52-58, 2017.

43. Pompili S, Sferra R, Gaudio E, Viscido A, Frieri G, Vetuschi A and Latella G: Can Nrf2 modulate the development of intestinal fibrosis and cancer in inflammatory bowel disease? Int J Mol Sci 20: 4061, 2019

44. Cai L, Tu L, Li T, Yang X, Ren Y, Gu R, Zhang Q, Yao H, Qu X, Wang Q and Tian J: Downregulation of lncRNA UCA1 ameliorates the damage of dopaminergic neurons, reduces oxidative stress and inflammation in Parkinson's disease through the inhibition of the PI3K/Akt signaling pathway. Int Immunopharmacol 75: 105734, 2019.

45. Liu AH, Chu M and Wang YP: Up-regulation of Trem 2 inhibits hippocampal neuronal apoptosis and alleviates oxidative stress in epilepsy via the PI3K/Akt pathway in mice. Neurosci Bull 35: 471-485, 2019.

46. Ouyang ZH, Wang WJ, Yan YG, Wang B and Lv GH: The PI3K/Akt pathway: A critical player in intervertebral disc degeneration. Oncotarget 8: 57870-57881, 2017.

47. Meierjohann S: Oxidative stress in melanocyte senescence and melanoma transformation. Eur J Cell Biol 93: 36-41, 2014.

This work is licensed under a Creative Commons Attribution-NonCommercial-NoDerivatives 4.0 International (CC BY-NC-ND 4.0) License. 